\title{
Cartographie et révolution numérique : la bibliothèque à l'ère électronique
}

\author{
Map-making and the Digital Révolution: The Library in the \\ Electronic Age \\ Cartografía y revolución digital: la biblioteca en la era \\ electrónica
}

\section{Jean-François Palomino}

Volume 47, numéro 3, juillet-septembre 2001

URI : https://id.erudit.org/iderudit/1032581ar

DOI : https://doi.org/10.7202/1032581ar

Aller au sommaire du numéro

Éditeur(s)

Association pour l'avancement des sciences et des techniques de la documentation (ASTED)

ISSN

0315-2340 (imprimé)

2291-8949 (numérique)

Découvrir la revue

Citer cet article

Palomino, J.-F. (2001). Cartographie et révolution numérique : la bibliothèque à l'ère électronique. Documentation et bibliothèques, 47(3), 119-122.

https://doi.org/10.7202/1032581ar
Résumé de l'article

À la faveur de la révolution numérique et de l'utilisation de plus en plus généralisée d'Internet, la cartographie se transforme dans ses modes de consultation, de diffusion et de conservation. Les projets et les réalisations de numérisation cartographique se multiplient au profit des spécialistes, des étudiants et du public en général. Des développements technologiques rapides permettent désormais de consulter des cartes de haute qualité. La Bibliothèque nationale du Québec offre près de 1500 cartes anciennes dans son site Web. Même si les standards restent à définir, la numérisation en vue de la conservation se répand. Le déclin de la production des cartes sur papier s'accélère et l'on favorise activement l'utilisation des données géographiques numérisées. Il importe cependant de mettre en place des mécanismes assurant la conservation de ces documents, comme plusieurs bibliothèques s'y emploient.
Tous droits réservés (c) Association pour l'avancement des sciences et des techniques de la documentation (ASTED), 2001
Ce document est protégé par la loi sur le droit d'auteur. L'utilisation des services d'Érudit (y compris la reproduction) est assujettie à sa politique d'utilisation que vous pouvez consulter en ligne. 


\title{
Cartographie et révolution numérique : la bibliothèque à l'ère électronique
}

\author{
Jean-François Palomino \\ Bibliothèque nationale du Québec
}

\begin{abstract}
À la faveur de la révolution numérique et de l'utilisation de plus en plus généralisée d'Internet, la cartographie se transforme dans ses modes de consultation, de diffusion et de conservation. Les projets et les réalisations de numérisation cartographique se multiplient au profit des spécialistes, des étudiants et du public en général. Des développements technologiques rapides permettent désormais de consulter des cartes de haute qualité. La Bibliothèque nationale du Québec offre près de 1500 cartes anciennes dans son site Web. Même si les standards restent à définir, la numérisation en vue de la conservation se répand. Le déclin de la production des cartes sur papier s'accélère et l'on favorise activement l'utilisation des données géographiques numérisées. Il importe cependant de mettre en place des mécanismes assurant la conservation de ces documents, comme plusieurs bibliothèques s'y emploient.
\end{abstract}

\section{Map-making and the Digital Revolution: The Library in the Electronic Age}

The digital revolution and the increasing use of the Internet have had an impact on the use of maps and their conservation. Specialists, students and the public in general are able to make use of digital maps and will benefit from projects that are under way. High-speed technology enables one to consult maps of a better quality. The Bibliothèque nationale du Québec has approximately 1,500 maps on its Web site. While standards have yet to be established, digitalisation as a means of conservation has increased. Fewer maps are being printed on paper and the use of digital geographic data is on the rise. However, means will have to be undertaken to conserve maps and several libraries are working on such means.

\section{Cartografía y revolución digital : la biblioteca en la era electrónica}

Con la revolución numérica y la utilización cada vez más generalizada de la Internet, la cartografía se transforma en cuanto a los modos de consulta, difusión y conservación. Los proyectos y las realizaciones de la digitalización cartográfica se multiplican para beneficio de los especialistas, estudiantes y público en general. Los desarrollos tecnológicos rápidos permiten desde ahora consultar mapas de alta calidad. La Biblioteca Nacional de Quebec ofrece casi 1500 mapas antiguos en su sitio Web. Aun si quedan por definirse los estándares, la conservación digitalizada se expande. El ocaso de la producción de mapas de papel se acelera y se prefiere mucho más el uso de datos geográficos digitalizados. No obstante, es importante implantar mecanismos que aseguren la conservación de estos documentos, tal como lo hacen muchas bibliotecas.
II n'y a plus de doute possible : nous vivons depuis quelques années une véritable révolution technologique, soutenue notamment par l'avènement du numérique et d'Internet. La plupart des acteurs culturels en sont bien conscients, à preuve les nombreux ouvrages et colloques entièrement consacrés aux nouveaux médias ou à l'impact des nouvelles technologies sur les différentes sphères de la connaissance. L'apparition de nouveaux supports et de nouveaux moyens de diffusion bouleverse nos façons d'agir, de penser et de percevoir le monde, un peu à l'image des répercussions de l'imprimerie au $X V I^{e}$ siècle.

Nos préoccupations ici concernent surtout l'écriture cartographique qui, comme le texte écrit, est en proie à une profonde mutation. De plus en plus utilisée sur support numérique, elle subit, elle aussi, cette dématérialisation qui n'est pas sans conséquence pour les producteurs et utilisateurs de cartes, ni pour tous ceux chargés de rassembler, de conserver et de diffuser l'information auprès du public. Les lecteurs de Documentation et bibliothèques ont été initiés dans un précédent numéro aux principes de base de la cartographie numérique ${ }^{1}$. En poursuivant dans cet article la réflexion déjà amorcée, notre intention est de cerner les principales conséquences pour les bibliothèques et leurs usagers du passage de la (re)production conventionnelle à la (re)production électronique. Deux points seront plus particulièrement examinés: la numérisation et la diffusion de collections déjà existantes et l'acquisition d'information cartographique numérique.

\section{Cartes anciennes: numériser pour diffuser et préserver}

Sans conteste, Internet est aujourd'hui un outil indispensable pour ceux qui cherchent la trace de cartes anciennes. En quelques années seulement, un nombre considérable d'institutions l'ont mis à profit pour améliorer leur visibilité et faire la promotion de leurs collections. Dans les années 1970, bibliothèques, centres d'archives et musées commencent l'informatisation des catalogues, des répertoires et des inventaires. Dans les années

1. Pierre Lépine. 1996. Géomatique et cartographie numérique ou de la disponibilité des systèmes d'information à référence spatiale. Documentation et bibliothèques $42 \mathrm{n}^{\circ} 2$ (avril-juin) : 87-92. 
1990, ces catalogues informatisés quittent leurs environnements locaux pour intégrer le plus vaste des réseaux, Internet. Les métadonnées y sont diffusées massivement, accessibles des bureaux et des foyers branchés, au grand bonheur des chercheurs qui peuvent repérer rapidement la documentation et s'éviter des déplacements bien inutiles ${ }^{2}$.

II y a plus encore. Internet favorise non seulement la diffusion des métadonnées, mais aussi la diffusion des reproductions en format numérique. Avec les perfectionnements rapides des techniques de numérisation, l'abaissement des coûts d'équipement et le financement des différents paliers des gouvernements, les projets de numérisation se font de plus en plus nombreux. À la fois œuvres scientifiques et œuvres d'art, les cartes anciennes sont en soi des documents historiques très attrayants, fréquemment exploités pour attirer l'attention du visiteur et mettre en valeur les collections. Les projets misent non seulement sur l'aspect esthétique, mais aussi et surtout sur l'importance des cartes anciennes pour l'enseignement et la recherche dans plusieurs disciplines, notamment la généalogie, la géographie historique, I'histoire de la cartographie, l'histoire environnementale, l'histoire urbaine, l'histoire culturelle, l'histoire des idées, etc.

La numérisation peut être exploitée dans le cadre d'expositions virtuelles. Plus que du matériel brut, le contenu est replacé dans un contexte historique particulier afin de joindre le plus large public possible. Les créateurs de tels sites fournissent une grille d'interprétation plus ou moins élaborée, une clé qui permet une meilleure interprétation et compréhension de la documentation. Ces sites offrent du matériel didactique intéressant pour l'enseignement de l'histoire et il est à souhaiter que la qualité du contenu s'améliore dans les prochaines années ${ }^{3}$.

Porteuse d'information par ailleurs introuvable, la carte géographique est une source historique bien utile au chercheur. C'est souvent dans les détails les plus anodins qu'on retrouve l'information désirée, répondant à des questionnements précis de toponymie ou de localisation géographique. On fait généralement appel aux cartes géographiques pour retracer et identifier immeubles, lots cadastraux, propriétaires terriens, villages, routes, chemins de fer, cours d'eau et autres élé- ments humains ou naturels du territoire. Quand on connaît l'amplitude des changements spatio-temporels au cours des deux derniers siècles, notamment en milieu urbain, on prend vite conscience de l'importance de tels témoins du passé.

Les premières images présentées sur le Web n'étaient guère intéressantes pour le chercheur puisqu'elles n'enregistraient pas avec finesse ces menus détails de la description cartographique. Pour rendre les tracés lisibles, il fallait restreindre la reproduction à un secteur puisque les cartes reproduites en entier étaient trop floues. D'importants développements technologiques ont tour à tour remédié au problème et avec le temps, les créateurs sont parvenus à présenter une imagerie numérique de bien meilleure qualité. Évidemment, les degrés de résolution plus élevés engendrent des fichiers beaucoup plus lourds qui doivent être compressés pour faciliter la manipulation. Le document cartographique étant lu selon un mode différent du texte écrit, sans direction ni sens fixe, il convient de le rendre accessible en son entier. Mais transposer sur un écran d'ordinateur ce qui se lit habituellement sur une feuille beaucoup plus grande peut facilement désorienter le lecteur. D'où l'importance des logiciels de visualisation adaptés à la consultation de cartes géographiques et présentant notamment des fonctions de «zoom » et de défilement.

Tous ces problèmes ont été réglés au fur et à mesure de leur apparition. Depuis, un grand nombre de projets ont été mis en branle dans différentes bibliothèques nationales ou universitaires, donnant accès par Internet à une partie substantielle des collections ${ }^{4}$. Tout en facilitant la diffusion de l'information géographique pour les chercheurs aguerris, les projets de numérisation ont aussi pour objectif la préservation de la documentation originale. II ne faut pas oublier que les cartes anciennes sont des documents fragiles, rares et parfois uniques. La numérisation est un mode de reproduction offrant une solution de rechange intéressante à la consultation des originaux sans être néfaste à l'état matériel des documents. La stabilité à long terme de l'image numérique n'est pas encore assurée. II y a toutefois gros à parier que la reproduction numérique supplantera d'ici quelques années la reproduction sur microformes. II reste encore à mettre au point des normes de standardisation assurant des reproductions de qualité, mais cela ne devrait guère tarder. L'option est d'ailleurs de plus en plus envisagée dans plusieurs centres de conservation ${ }^{5}$.

À la Bibliothèque nationale du Québec, une grande portion de la collection de cartes anciennes (près de 1500 cartes) a déjà été numérisée pour des fins de diffusion dans Internet. Voilà un début prometteur et bien apprécié des usagers en quête de renseignements sur l'histoire et la géographie du Québec. II ne faut pas

2. Pour repérer les collections de cartes les plus pertinentes, on peut consulter différents répertoires. Le plus complet étant Olivier Loiseaux, (dir.), World Directory of Map Collections, Munich, K. G. Saur, 2000. Par ailleurs, on peut trouver, sur le site Oddens <http //oddens.geog.uu. nl/index.html>, une liste par pays des différentes collections de cartes dans Internet. Il est recommandé par ailleurs de lancer une recherche en interrogeant des catalogues collectifs tels Amicus (accès gratuit: <http ://amicus.nlc-bnc.ca/aaweb/amiloginf.htm $>$ ) et WorldCat (accès payant: <http://newfirstsearch.oclc.org/>). Plusieurs institutions québécoises possédant des cartes anciennes mettent à la disposition des usagers leur catalogue en ligne, que ce soit à la Bibliothèque nationale du Québec <http://www2.biblinat.gouv.qc.ca/>, à l'Université Laval (base de données de la cartothèque: <http://www.bibl.ulaval.ca/bd/carto/>), à l'Université McGill <http ://muse.mcgill.ca $>$. On pourra également trouver des cartes anciennes du territoire québécois dans plusieurs institutions étrangères, notamment à la Bibliothèque nationale de France (BN-Opaline : $<\mathrm{http} / /$ opaline.bnf.fr/>), à la Bibliothèque du Congrès <http :// catalog.loc.gov/> ou à la Newberry Library <http:// www.newberry.org/n1/collections/virtua.html $>$.

3. Jusqu'à présent, les seules expositions de cartes touchant le territoire québécois ont été réalisées par les Archives nationales du Canada (voir «Le Canada à l'échelle : les cartes de notre histoire ») <http://www.archives.ca/05/ 0514_f.html>, «Mémoire vivante »<http ://www.archives.ca/05/0509_f.html> et «Aux sources de la Nouvelle-France»<http $/ /$ www.archives.ca/05/0517_f.html $>$ et l'Université Laval <http://www.bibl.ulaval.ca/ress/ carto $2 /$ cadres.html $>$. Pour une liste étoffée des expositions de cartes anciennes dans Internet, voir: «Exhibitions » du site Map history <http://ihr.sas.ac.uk/maps/ webtexts.html\#america>.

4. Hormis le site de la Bibliothèque nationale du Québec évoqué plus haut, mentionnons celui de la Bibliothèque du Congrès, réalisation remarquable présentant plusieurs milliers de cartes organisées par thèmes tels que «Découvertes et explorations », "Transports et communications », «Villes et villages », « Campagnes militaires », etc. <http://lcweb2.loc.gov/ammem/gmdhtml/ gmdhome.html $>$. Voir également les sites de l'Université McGill <http ://imago.library.mcgill.ca/pugsley/>, de McMaster University <http ://www.mcmaster.ca/library/ maps/rchome.htm>, de l'Université du Texas <http:// www.lib.utexas.edu/maps/historical/index.html $>$, du collectionneur privé David Rumsey (plus de 5000 cartes : $<$ http $: / / w w w . d a v i d r u m s e y . c o m / i n d e x . a s p>$ ) et de l'Université de Toronto <http://www.library.utoronto.ca/ maplib/gta/main.html $>$. Pour une liste exhaustive des collections numériques disponibles dans Internet, voir la page «Image Sites» du site Map history <http ://ihr sas.ac.uk/maps/webimages.html>.

5. Sur ce sujet, voir entre autres Maxine K. Sitts, (ed.) Handbook for digital projects : a management tool for preservation and access. Andover: Northeast Document Center 2000. <http $/ / /$ nedcc.org/digital/dighome. htm >. 
cependant ralentir la cadence. À un moment où bibliothèques, centres d'archives et universités mettent de plus en plus en commun leurs ressources pour développer des projets de numérisation ${ }^{6}$, on ne peut qu'applaudir à la création de tels projets pour le territoire québécois. Ceux qui cherchent, par exemple, des traces cartographiques du temps de la NouvelleFrance doivent visiter plusieurs institutions au Québec, au Canada, aux États-Unis, en France ou en Angleterre. La dispersion de la documentation dans différentes institutions souvent très éloignées les unes des autres complique les démarches des chercheurs. Dans ce contexte, le numérique et Internet pourraient être fructueusement exploités pour faciliter le repérage, l'organisation et la diffusion de l'information cartographique. C'est pourquoi les partenariats entre différentes institutions québécoises ou même étrangères devront être sérieusement envisagés dans un très proche avenir.

\section{Cartes contemporaines: rassembler aujourd'hui pour les chercheurs de demain}

Qu'en est-il des cartes contemporaines ou cartes courantes? Les techniques informatiques ont, là aussi, complètement bouleversé la donne en automatisant de a à $z$ le métier de cartographe. La vitesse de production a ainsi rapidement décuplé, les tracés étant maintenant plus précis et plus efficacement mis à jour. De nombreux outils ont été créés dans le but de simplifier les opérations, par exemple des bases de données à référence spatiale qui conservent l'information cartographique pouvant être imprimée en temps voulu.

Avec le temps, l'automatisation est devenue si efficace que l'écart allait toujours grandissant entre la quantité d'information stockée et la quantité très limitée d'information imprimée. Malgré les progrès de l'équipement et des techniques d'impression, le tirage papier est demeuré trop coûteux pour les producteurs. Pour remédier au problème, la solution a été bien simple: distribuer l'information cartographique en format numérique à des utilisateurs qui ont la formation et les outils nécessaires pour l'exploiter sous cette forme. C'est une disci- pline toute nouvelle qui a été fondée à la suite de cette idée: la géomatique.

En quelques années seulement, ce champ disciplinaire institue ses propres lieux de formation et de recherche aux niveaux collégial et universitaire. Plusieurs cartothèques doivent s'adapter au nouveau contexte. Quelques-unes se transforment en centres d'information géographique. Jadis, il n'avait jamais été très difficile de suivre l'évolution de la production cartographique pour maintenir ses collections à jour. Maintenant, pour continuer à répondre aux besoins des chercheurs, plusieurs cartothécaires doivent aussi revêtir le manteau de spécialistes en SIG (Systèmes d'information géographique), une grande partie des tâches étant dévolue au repérage et à l'acquisition de publications électroniques.

La mutation radicale qu'on a prédite se fait encore attendre. II s'écoulera sûrement beaucoup de temps avant que la "e-carte » se pose en substitut définitif de la carte papier. Vu les avantages manifestes d'un tel format sur le numérique, la cohabitation promet d'être longue.

II faut cependant admettre que le déclin de la production traditionnelle est fulgurant. On s'en rend compte simplement en examinant la cartographie des différentes agences gouvernementales ou municipales. Parmi maints exemples, retenons celui de l'Atlas national du Canada, publié depuis 1906 en format papier. La sixième édition de cet ouvrage de référence majeur n'est désormais disponible qu'en format numérique dans Internet <http://atlas.gc. $\mathrm{ca} />$. Le gouvernement fédéral a mis aussi à la disposition des internautes des données topographiques numériques libres de droit, couvrant une partie du territoire à l'échelle 1:50 $000<$ http://toporama.cits. rncan.gc.ca/) $>>7$. Aux États-Unis, une compagnie privée s'est chargée de mettre gratuitement en ligne les cartes topographiques du United States Geologic Survey (USGS), principale agence de production cartographique au pays <http://topozone. com>. Pour ce qui est du Québec, rien encore n'est disponible dans Internet. Comme la Photocartothèque québécoise doit s'autofinancer, elle diffuse, sur demande et à titre onéreux, l'information géographique qu'elle produit <http://www. mrn.gouv.qc.ca/photocartotheque/s. Auparavant, chaque carte était rééditée quand le nombre de modifications le justifiait. Aujourd'hui, il n'y a plus de système d'édition qui tient, les cartes étant mises à jour à une fréquence trop élevée. À la ville de Montréal, le Service d'urbanisme se chargeait de produire, depuis les années 1950 , des plans à très grande échelle (entre 1:600 et 1:2400), montrant tous les îlots, rues et bâtiments de la ville. Au début des années 1990, on a complètement cessé la production de tels plans. Depuis, c'est la Division de la géomatique qui gère et garde à jour dans une géo-base les données concernant le cadre bâti de la ville, les périmètres des propriétés, le rôle foncier, le rôle locatif, etc.

Tous les gestionnaires du territoire ont ou auront bientôt leurs bases de données géographiques à référence spatiale. C'est là une source d'information inestimable, méconnue du public, mais souvent produite grâce aux deniers publics. Une part importante de l'information cartographique s'y trouve, distribuée uniquement en format numérique, que ce soit par cédérom, courriel, Internet ou téléchargement FTP.

Actuellement, les instances publiques tentent de susciter une demande pour cette information. À cet égard, quelques programmes de subventions ont été mis en place (surtout au niveau fédéral) pour stimuler l'utilisation des données géographiques $^{8}$. II va de soi que les bibliothèques jouent un rôle de premier plan dans ces opérations visant à démocratiser l'accès à l'information géographique.

L'ajustement des bibliothèques doit se faire à tous les niveaux. II reste, par exemple, à modifier la Description bibliographique internationale normalisée des documents cartographiques ISBD(CM) pour inclure les cartes électroniques ${ }^{9}$.

6. En Écosse, la Bibliothèque nationale d'Écosse et l'Université d'Édimbourg ont conjointement entrepris de numériser les cartes d'Écosse, manuscrites ou publiées, réalisées entre 1590 et 1740 . Par le fait même, les participants ont entrepris de compléter le catalogage de cette documentation qui comprend aussi les archives textuelles liées aux documents cartographiques et permettant de mieux les comprendre.

7. Ce portail présente des représentations à basse résolution des produits en vente. Les images ne remplacent pas la carte topographique conventionnelle. Il a toutefois le noble objectif d'offrir des données qui s'adressent à un large éventail d'usagers tout en rendant la géomatique accessible à tous.

8. À cet égard, l'un des sites les plus remarquables est Géogratis, un site Web couplé à un site FTP permettant la distribution de données géospatiales canadiennes. Les données sont disponibles pour téléchargement sans frais <http://geogratis.cgdi.gc.ca/>.

9. Voir en ce sens, la proposition suivante présentement à l'étude: Proposals for a revision to include electronic re sources $<$ http ://ifla.inist.fr/VII/s6/pubs.htm>. 
Pour l'instant, ces documents reçus sur cédéroms sont décrits selon les règles de l'ISBD(ER) qui exclut un certain nombre d'éléments nécessaires à l'identification et la description des documents cartographiques.

Le problème le plus préoccupant est celui de la préservation à long terme de l'information cartographique numérique. Les concepteurs et producteurs de cartes pensent et agissent en fonction des besoins immédiats, et cela est tout à fait normal. Les nouveaux systèmes informatiques assurent une mise à jour permanente qui garantit leur efficacité. Mais une mise à jour efficace signifie également une plus grande quantité d'information périmée, inutile, voire inutilisable pour certains usagers, mais certainement très profitable pour d'autres chercheurs intéressés par l'évolution spatio-temporelle du territoire. Nul besoin d'en faire la démonstration: la carte géographique est une source d'information sans équivalent pour qui sait l'exploiter. Historiens, géographes, archéologues, environnementalistes, urbanistes, ingénieurs et autres chercheurs connaissent l'importance des cartes anciennes pour trouver quelques traces du passé. Plusieurs bibliothèques mettent ainsi à leur disposition des suites cartographiques permettant de connaître le territoire à intervalles plus ou moins réguliers.

Avec le déclin de la production papier, le risque est grand de voir ces suites s'éteindre. Les producteurs ne semblent pas soucieux de garder copie de l'information périmée. Si cette information n'est pas conservée, si aucune instance ne juge bon de garder une copie des changements du territoire, quelle matière resterat-il aux chercheurs de demain qui voudront connaître l'environnement dans lequel nous vivons aujourd'hui? Que restera-t-il à ceux qui voudront connaître, dans quinze, trente, cinquante ou cent ans, l'évolution du tissu urbain, la localisation des entreprises polluantes alors disparues, la répartition socio-économique dans l'espace, l'année de construction de tel ou tel bâtiment ? La disparition des matériaux de base empêchera de mener à bien ces études. Un trou béant menace les chercheurs des générations futures si aucune action concrète n'est engagée pour remédier à la situation. D'où l'importance de bien penser ce que nous pouvons aujourd'hui conserver pour les besoins de demain.
C'est une question de prévoyance et d'intégrité.

Quelques institutions étrangères ont déjà fait face au problème. En Angleterre par exemple, une entente a été conclue entre l'Ordnance Survey (agence gouvernementale productrice de cartes) et les différentes bibliothèques dépositaires, dont la British Library. Chaque année, un cliché des bases de données cartographiques est pris et déposé dans ces bibliothèques. Les bases de données peuvent être consultées par les usagers, mais il est strictement défendu d'en télécharger le contenu.

De telles dispositions devraient guider la Bibliothèque nationale du Québec dans ses prochaines démarches. L'importance patrimoniale de la documentation cartographique a été une première fois attestée par l'instauration du dépôt légal des cartes, en 1980. Avec le dépôt légal des publications électroniques mis en place en 1992, la Bibliothèque s'est dotée d'un outil permettant le développement de collections plus représentatives du patrimoine documentaire québécois. Mais il ne suffit pas d'être chargé de l'application du Règlement sur le dépôt légal pour mettre en branle des projets de longue haleine. II faut avant tout savoir sensibiliser les producteurs de données cartographiques au problème de la préservation de la documentation numérique. Il faut également disposer des ressources humaines et matérielles adéquates. Internet ne vient certainement pas concrétiser les vieux rêves utopiques de gestionnaires, c'est-à-dire remplacer l'homme par la machine. Les nouvelles technologies produisent du contenu toujours plus imposant en quantité et en qualité. La gestion de documents spécialisés demande un personnel tout aussi spécialisé sachant bien s'adapter aux nouvelles réalités pour rassembler, ordonner et diffuser l'information.

Demeurer à la fine pointe des technologies de l'information s'avère très souhaitable, mais n'est certainement pas une fin en soi. En pensant la «bibliothèque du futur », il ne faut pas perdre de vue les missions fondamentales qu'elle s'est données dans les siècles passés: conserver le savoir des hommes pour les générations futures et faire connaître ce patrimoine au plus grand nombre possible. 